The University of San Francisco

USF Scholarship: a digital repository @ Gleeson Library |

Geschke Center

Physics and Astronomy

College of Arts and Sciences

11-12-2001

\title{
Quantum Anomaly in Molecular Physics
}

Horacio E. Camblong

University of San Francisco, camblong@usfca.edu

Follow this and additional works at: http://repository.usfca.edu/phys

Part of the Physics Commons

\section{Recommended Citation}

Camblong, Horacio E., "Quantum Anomaly in Molecular Physics" (2001). Physics and Astronomy. Paper 2.

http://repository.usfca.edu/phys/2

This Article is brought to you for free and open access by the College of Arts and Sciences at USF Scholarship: a digital repository @ Gleeson Library | Geschke Center. It has been accepted for inclusion in Physics and Astronomy by an authorized administrator of USF Scholarship: a digital repository @ Gleeson Library | Geschke Center. For more information, please contact repository@usfca.edu. 


\title{
Quantum Anomaly in Molecular Physics
}

\author{
Horacio E. Camblong, ${ }^{1}$ Luis N. Epele, ${ }^{2}$ Huner Fanchiotti, ${ }^{2}$ and Carlos A. García Canal ${ }^{2}$ \\ ${ }^{1}$ Department of Physics, University of San Francisco, San Francisco, California 94117-1080 \\ ${ }^{2}$ Laboratorio de Física Teórica, Departamento de Física, Universidad Nacional de La Plata, C.C. 67-1900 La Plata, Argentina
}

(Received 15 June 2001; published 12 November 2001)

\begin{abstract}
The interaction of an electron with a polar molecule is shown to be the simplest realization of a quantum anomaly in a physical system. The existence of a critical dipole moment for electron capture and formation of anions, which has been confirmed experimentally and numerically, is derived. This phenomenon is a manifestation of the anomaly associated with quantum symmetry breaking of the classical scale invariance exhibited by the point-dipole interaction. Finally, analysis of symmetry breaking for this system is implemented within two different models: point dipole subject to an anomaly and finite dipole subject to explicit symmetry breaking.
\end{abstract}

DOI: $10.1103 /$ PhysRevLett.87.220402

PACS numbers: 03.65.Ge, 11.30.-j, 31.10.+z, 31.15.-p

In this Letter we establish the existence of a remarkably simple physical realization of a quantum anomaly in $\mathrm{Na}-$ ture. An anomaly $[1,2]$ is one of the three possible types of symmetry breaking exhibited by a physical system with an invariance of some kind - the other two being explicit and spontaneous symmetry breaking [3]. In particular, it arises when a classical invariance of a system is violated upon quantization. Its physical realizations in Nature have been recognized in high-energy physics since the introduction of the Adler-Bell-Jackiw anomaly [4-6], which amounts to violation of chiral symmetry. As a practical tool, this concept has become useful for the analysis of elementary particles in the standard model [2] and its extensions [7], as well as in string theory [8]. In addition to its phenomenological relevance, the study of anomalies has been the source of numerous theoretical investigations [1]; for example, (i) in the path-integral formulation, the chiral-symmetry-breaking anomaly is due to lack of invariance of the functional measure [9]; (ii) in the Hamiltonian formulation, an operator becomes anomalous when it does not keep invariant the domain of definition of the Hamiltonian [10].

The novel physical realization of a quantum anomaly that we now consider occurs in the realm of molecular physics. Specifically, while the consequences implied by our analysis have been known in the literature for some time now, their interpretation in terms of the anomaly concept is totally new. Therefore, in this Letter, by studying this particular realization, we highlight the relevance and simplicity of the anomaly phenomenon beyond its original high-energy physics context. In fact, this remarkable interpretation is the central result of our Letter.

Our analysis involves three essential ingredients: (i) the identification of the relevant symmetry that undergoes an anomalous breaking; (ii) the renormalization, including dimensional transmutation [11,12], of a nonrelativistic inverse square potential; and (iii) the subsequent application of the inverse square potential to the interaction of an electric dipole with a charge.
First, the symmetries relevant to our work are identical to those of the inverse square potential; under time reparametrizations, they include scale invariance [13], which is part of a larger conformal symmetry with $\mathrm{SO}(2,1)$ group-theory structure [14]. Surprisingly, the relevance of these symmetry analyses for the dipole potential has remained unnoticed despite its central physical importance in molecular physics. Correspondingly, in this Letter, we review and adapt the symmetry arguments to the case of the dipole-charge interaction.

Second, we have recently considered the inverse square potential using field-theory techniques and shown the existence of a critical coupling for the onset of dimensional transmutation [15]. On the other hand, dimensional transmutation has been recently viewed as an example of a quantum anomaly: that associated with the breaking of scale invariance $[16,17]$. However, this anomaly has been discussed only in the context of the two-dimensional deltafunction potential [18]. In this Letter we show that the concepts of anomaly and dimensional transmutation apply to the inverse square potential and related physical problems, including the intriguing electric dipole-charge interaction, with far-reaching implications in molecular physics.

Third, the nonrelativistic interaction of an electric dipole with a charge poses a problem whose physical relevance was first recognized in nuclear physics $[19,20]$ and then in molecular physics [21-23]. In the traditional treatments of this problem [24], a key ingredient is the existence of a critical dipole moment for the capture of the electric charge by the dipole. This unambiguous prediction of quantum mechanics has led to a standard lore in molecular physics, according to which any neutral molecule with a dipole moment of the order of 2D or greater [25] should be capable of capturing an electron and sustaining a molecular anion [26]. Recent experiments [27,28] and numerical simulations [28] confirm this generic prediction. This immediately leads one to pose the question: what is the origin of this critical coupling and why is it robust? In this Letter we shed light on this issue by treating the problem using 
scale invariance and its associated anomaly, dimensional transmutation.

The interaction of an electric charge $Q$ with a point dipole $\mathbf{p}$, which we treat nonrelativistically and refer to as the point-dipole potential, is described by means of

$$
V(\mathbf{r})=K_{e} \frac{Q p \cos \theta}{r^{2}},
$$

which can be regarded as a generalized inverse square potential with an anisotropic coupling strength. As usual, in Eq. (1), the polar angle $\theta$ is measured from the direction of the dipole moment, and we assume that the problem occurs in ordinary three-dimensional space. The coupling can be rewritten in a dimensionless form

$$
\lambda=-\frac{2 m K_{e}}{\hbar^{2}} Q p=\frac{p}{p_{0}},
$$

with $m$ being the reduced mass of the system and $K_{e}$ the electrostatic constant. In Eq. (2) the characteristic dipole moment $p_{0}$ sets the scale for our analysis and is the relevant dimensional parameter whose order of magnitude will provide an estimate for criticality (charge capture). For the particular case of an electron interacting with a polar molecule, $Q=-e$ (with $e$ being the electron charge magnitude), and $p_{0}=e a_{0} / 2 \approx 1.271 D$, where $a_{0}$ is the Bohr radius and $D$ is the debye [25]. The Hamiltonian associated with Eqs. (1) and (2),

$$
H=-\frac{\hbar^{2}}{2 m}\left[\nabla^{2}+\lambda \frac{\cos \theta}{r^{2}}\right],
$$

is explicitly scale and conformally invariant, as the analysis below shows.

The corresponding classical Lagrangian $L=m v^{2} / 2-$ $V(\mathbf{r})$ has an associated action that is invariant under the scale transformations $t \rightarrow \tau t, \mathbf{r} \rightarrow \varrho \mathbf{r}$ (with $\tau>0$ and $\varrho>0), \varrho^{2}=\tau$; this property is shared by the larger class of homogeneous potentials of degree -2 [12], which also includes the two-dimensional delta-function potential. In the language of dimensional analysis, this symmetry means that the point-dipole potential has no characteristic dimensional scales and the coupling $\lambda$ is dimensionless [29].

The symmetry analysis under time reparametrizations can be generalized to [30,31] $t \rightarrow \tilde{t}=t-\alpha f(t), \mathbf{r} \rightarrow$ $\tilde{\mathbf{r}}(\tilde{t})=J^{\delta} \mathbf{r}(t)$, with $J=|d \tilde{t} / d t|$. Invariance of the action occurs only when the following two conditions are simultaneously satisfied: (i) $\delta=1 / 2$ and (ii) $f(t)$ is quadratic in $t$. This selects the $\mathrm{SO}(2,1)$ conformal group, just as for the inverse square potential [14], the magnetic monopole [30], and the magnetic vortex [31], with the following three generators: (i) the Hamiltonian $H$, Eq. (3), associated with time translations $t \rightarrow t-\alpha$ [for $f(t)=1]$; (ii) the dilation generator

$$
D=t H-\frac{1}{4}(\mathbf{r} \cdot \mathbf{p}+\mathbf{p} \cdot \mathbf{r}),
$$

associated with the scale transformation defined in the previous paragraph, with $\tau=1-\alpha$ [for $f(t)=t$ ]; and (iii) the conformal generator

$$
K=H t^{2}-\frac{1}{2}(\mathbf{p} \cdot \mathbf{r}+\mathbf{r} \cdot \mathbf{p}) t+\frac{1}{2} m r^{2},
$$

associated with the time special conformal transformation $1 / t \rightarrow 1 / t+\alpha$ [for $\left.f(t)=t^{2}\right]$. The corresponding commutators $[D, H]=-i \hbar H,[D, K]=i \hbar K$, and $[H, K]=2 i \hbar D$ show that these three operators form an $\mathrm{SO}(2,1)$ algebra [32].

We will now examine how scale invariance is broken at the quantum-mechanical level. This symmetry breaking manifests itself in the appearance of a critical dipole moment, whose existence and numerical value we will establish next by generalizing our treatment of the inverse square potential [15]. This goal can be accomplished by writing the Schrödinger equation for the point dipole in spherical coordinates, with the separation of variables $\Psi(r, \theta, \phi)=u(r) \Theta(\theta) e^{i m \phi} / r$, where the azimuthal dependence corresponds to conservation of the axial component $L_{z}$ of angular momentum. Then, the corresponding equations for $r$ and $\theta$ are explicitly given by

$$
\frac{d^{2} u(r)}{d r^{2}}+\left(\eta+\frac{\gamma}{r^{2}}\right) u(r)=0
$$

and

$$
\hat{A} \Theta(\theta)=\gamma \Theta(\theta),
$$

where $\eta=2 m E / \hbar^{2}$,

$$
\hat{A}=-\Lambda^{2}+\lambda \cos \theta,
$$

and $\Lambda^{2}=L^{2} / \hbar^{2}$ is the dimensionless angular momentum (squared). Equations (6) and (7) constitute a coupled system of eigenvalue equations linked by the separation constant $\gamma$. Notice that Eq. (6) can be interpreted as defining an isotropic inverse square potential for the zero angular-momentum channel in three dimensions, with coupling strength $\lambda_{\text {ISP }} \equiv \gamma$. In addition, $\gamma$ is implicitly related to the actual coupling $\lambda$ of the point-dipole potential by means of the eigenvalue equation (7). This relation can be obtained by recasting Eq. (7) into matrix form through the matrix

$$
M(\gamma, \lambda)=-A(\lambda)+\gamma \mathbb{1}
$$

(where $\mathbb{1}$ is the identity matrix) and subsequently setting up the corresponding characteristic equation [21-23]

$$
D(\gamma, \lambda) \equiv \operatorname{det} M(\gamma, \lambda)=0 .
$$

It should be noticed that the existence of symmetry breaking for the dipole potential can be viewed as a consequence of the corresponding symmetry breaking for the inverse square potential. As shown in Ref. [15], this quantum anomaly occurs for the inverse square potential in the supercritical or strong-coupling regime $\gamma \geq \gamma^{(*)}=1 / 4$ (for $l=0$ in three dimensions). It then follows that there exists a critical value $\lambda^{(*)}$ of the dimensionless dipole moment to be determined from the corresponding critical inverse square coupling $\gamma^{(*)}=1 / 4$, i.e., 


$$
D\left(\gamma^{(*)}, \lambda^{(*)}\right)=0,
$$

and such that a quantum anomaly occurs for the strongcoupling regime $\lambda \geq \lambda^{(*)}$. A straightforward calculation shows that $\lambda^{(*)} \approx 1.279$, which amounts to the familiar critical dipole moment $p^{(*)} \approx 1.625 D$ [19-23]. Extensive empirical and numerical studies have confirmed the existence of a critical dipole moment of a similar value for a large number of molecules [27,28]. This remarkable universal property of polar molecules can be regarded as the simplest physical example of a quantum anomaly.

The fact that the predictions arising from the quantum anomaly analyzed in the previous paragraph agree with the corresponding empirical and numerical findings requires further elaboration. In effect, a polar molecule is better modeled as a finite dipole, with an interaction potential

$$
V(\mathbf{r})=K_{e} Q q\left(\frac{1}{R_{+}}-\frac{1}{R_{-}}\right)=K_{e} \frac{Q p \cos \theta}{r^{2}}+V_{\mathrm{sb}}(\mathbf{r}) .
$$

In Eq. (12) $R_{ \pm}$represents the distance to the charge $Q$ from the positive and negative charges of the dipole, which are separated by a distance $a$. Equation (12) displays the point-dipole potential (1), with $p=q a(l=1)$, supplemented by a symmetry-breaking potential $V_{\mathrm{sb}}(\mathbf{r})$, which includes higher-order multipoles (for $l>1$ and $r>a / 2$, with moments $\sim q a^{l}=p a^{l-1}$ ), as well as the contribution to the potential for $r<a / 2$. In short, in this model, the $\mathrm{SO}(2,1)$ symmetry of potential (1) undergoes explicit symmetry breaking by the introduction of additional terms in the Hamiltonian. The corresponding Schrödinger equation with potential (12) can be derived by separation of variables in prolate spheroidal coordinates [33], thus providing a solution [21-23,34] that illustrates the effect of adding explicit symmetry-breaking terms.

A priori, it is by no means obvious that the approximate point-dipole representation captures the correct behavior and the correct numerical value of the critical dipole moment. However, as we will show next, this is indeed the case. In other words, even though the finite dipole introduces a length scale $a$ and amounts to an example of explicit symmetry breaking, the existence of a critical dipole moment as well as its numerical value are independent of $a$. In fact, this result is confirmed by the explicit solution of the problem with potential (12). In short, the simplified point-dipole model exhibits an anomaly, whose relevance is highlighted by a robust prediction - one that survives when the finite size of the molecule is considered.

Let us now see the dimensional argument that proves the statement of the previous paragraph. The characteristic dimensional parameters for the dynamics of the finite dipole are $\hbar, m, q$, and $a$, as well as the finite charge $Q$; moreover the interaction involves only the product $Q q$. Then, according to Buckingham's Pi theorem of dimensional analysis [35],

$$
E_{\mathrm{gs}}=-\frac{\hbar^{2}}{2 m a^{2}} F(\lambda)
$$

where $\lambda$ is the dimensionless combination of the given parameters that we previously defined in Eq. (2) and $F(\lambda)$ is an arbitrary function of $\lambda$. On the other hand, the critical value of the dimensionless coupling, $\lambda^{(*)}$, occurs when the ground state energy $E_{\mathrm{gs}}$ comes into existence going through a zero value [36]. Thus, the critical dipole moment is defined by the condition

$$
F\left(\lambda^{(*)}\right)=0
$$

whose solution is a dimensionless number independent of the size $a$ of the dipole. In particular, the critical value survives in the limit $a \rightarrow 0$, which amounts to the ideal point dipole. This shows that the point-dipole model with anomalous symmetry breaking predicts the correct physics of the finite dipole, for which the symmetry is explicitly broken.

In conclusion, we have found theoretical and empirical evidence-further confirmed by numerical computations - of the existence of a quantum anomaly in molecular physics. Specifically, this anomaly is manifested by the formation of anions through electron capture by polar molecules with supercritical dipole moments and, to our knowledge, represents the simplest realization of quantum-mechanical symmetry breaking in a physical system.

This research was supported in part by CONICET and ANPCyT, Argentina (L.N.E., H.F., and C. A. G. C.) and by the University of San Francisco Faculty Development Fund (H.E.C.). Instructive discussions with Professor Carlos R. Ordóñez and Professor B. Montgomery Pettitt are gratefully acknowledged by H. E. C.

[1] S. B. Treiman, R. Jackiw, B. Zumino, and E. Witten, Current Algebras and Anomalies (World Scientific, Singapore, 1985).

[2] J. F. Donoghue, E. Golowich, and B. R. Holstein, Dynamics of the Standard Model (Cambridge University Press, Cambridge, U.K., 1992).

[3] An introductory discussion of the three types of symmetry breaking, with elementary examples of each kind, can be found in B. R. Holstein, hep-ph/0010033.

[4] J. S. Bell and R. Jackiw, Nuovo Cimento A 60, 47 (1969). Also see R. Jackiw, in Lectures on Current Algebra and Its Applications (Princeton University Press, Princeton, 1972).

[5] S. Adler, Phys. Rev. 177, 2426 (1969). Also see S. Adler, in Lectures on Elementary Particles and Quantum Field Theory-1970 Brandeis University Summer Institute in Theoretical Physics, edited by S. Deser, M. Grisaru, and H. Pendleton (MIT Press, Cambridge, MA, 1970), Vol. 1.

[6] S. Adler and W. A. Bardeen, Phys. Rev. 182, 1517 (1969).

[7] L. N. Epele, C. A. García Canal, and W. A. Ponce, Phys. Lett. B 411, 159 (1997), and references therein.

[8] J. Polchinski, String Theory (Cambridge University Press, Cambridge, U.K., 1998), Vols. I and II.

[9] K. Fujikawa, Phys. Rev. Lett. 42, 1195 (1979); Phys. Rev. D 21, 2848 (1980). 
[10] J. G. Esteve, Phys. Rev. D 34, 674 (1986).

[11] S. Coleman and E. Weinberg, Phys. Rev. D 7, 1888 (1973).

[12] H. E. Camblong, L. N. Epele, H. Fanchiotti, and C. A. García Canal, Ann. Phys. (N.Y.) 287, 14 (2001).

[13] R. Jackiw, Phys. Today 25, No. 1, 23 (1972).

[14] V. de Alfaro, S. Fubini, and G. Furlan, Nuovo Cimento Soc. Ital. Fis. 34A, 569 (1976).

[15] H. E. Camblong, L. N. Epele, H. Fanchiotti, and C. A. García Canal, Phys. Rev. Lett. 85, 1590 (2000); Ann. Phys. (N.Y.) 287, 57 (2001).

[16] R. Jackiw, in M. A. B. Bég Memorial Volume, edited by A. Ali and P. Hoodbhoy (World Scientific, Singapore, 1991).

[17] B. R. Holstein, Am. J. Phys. 61, 142 (1993).

[18] In addition to the work of Refs. [16,17], the interpretation of dimensional transmutation as an anomaly is critically revisited in A. Cabo, J. L. Lucio, and H. Mercado, Am. J. Phys. 66, 240 (1998). However, their analysis - limited to the scattering solutions - misses relevant information conveyed by the bound-state sector of the theory.

[19] E. Fermi and E. Teller, Phys. Rev. 72, 406 (1947).

[20] A. S. Wightman, Phys. Rev. 77, 521 (1949).

[21] J.-M. Lévy-Leblond, Phys. Rev. 153, 1 (1967).

[22] W. Byers Brown and R. E. Roberts, J. Chem. Phys. 46, 2006 (1967).

[23] O. H. Crawford, Proc. Phys. Soc. London 91, 279 (1967).

[24] The early history of this problem is discussed in J.E. Turner, Am. J. Phys. 45, 758 (1977).

[25] The debye $D$ is a characteristic scale for molecular dipole moments, defined to be exactly $1 D=10^{-18}$ esu-cm.

[26] O. H. Crawford, Mol. Phys. 20, 585 (1971); O. H. Crawford and W. R. Garrett, J. Chem. Phys. 66, 4968 (1977).

[27] R. D. Mead, K. R. Lykke, and W. C. Lineberger, J. Chem. Phys. 81, 4883 (1984); K. R. Lykke, D. M. Neumark, T. Andersen, V. J. Trappa, and W. C. Lineberger, J. Chem.
Phys. 87, 6842 (1987); A. S. Mullin, K. K. Murray, C. P. Schulz, and W. C. Lineberger, J. Phys. Chem. 97, 10281 (1993).

[28] C. Desfrançois, H. Abdoul-Carime, N. Khelifa, and J. P. Schermann, Phys. Rev. Lett. 73, 2436 (1994), and references therein.

[29] This property implies that, quantum-mechanically and for a sufficiently strong potential, the Hamiltonian fails to be bounded from below and is not self-adjoint, despite its Hermitian character. A relevant example of the application of the technique of self-adjoint extensions can be found in Ref. [16], where it is applied to the two-dimensional delta-function potential. This point is related to Ref. [10].

[30] R. Jackiw, Ann. Phys. (N.Y.) 129, 183 (1980).

[31] R. Jackiw, Ann. Phys. (N.Y.) 201, 83 (1990).

[32] B. Wybourne, Classical Groups for Physicists (Wiley, New York, 1974).

[33] The finite dipole is a particular case of the general twocenter problem presented in W. G. Baber and H. R. Hassé, Proc. Cambridge Philos. Soc. 31, 564 (1935).

[34] R. F. Wallis, R. Herman, and H. W. Milnes, J. Mol. Spectrosc. 4, 51 (1960); M. H. Mittleman and V. P. Myerscough, Phys. Lett. 23, 545 (1966); J. E. Turner and K. Fox, Phys. Lett. 23, 547 (1966); C. A. Coulson and M. Walmsley, Proc. Phys. Soc. London 91, 31 (1967); J. E. Turner, V. E. Anderson, and K. Fox, Phys. Rev. 174, 81 (1968); D. I. Abramov and I. V. Komarov, Teor. Mat. Fiz. 13, 209 (1972).

[35] E. Buckingham, Phys. Rev. 4, 345 (1914); P. W. Bridgman, Dimensional Analysis (Yale University Press, New Haven, 1922).

[36] The rapid growth in the value of $\left|E_{\mathrm{gs}}\right|$ in the molecular system, as $\lambda$ is increased beyond $\lambda^{(*)}$, corresponds to the well-known arbitrariness in the value of the anomalous scale in the renormalized theory [15]. 